\title{
Numerical Analysis of the Heat Sink Effect in the Infrared Inspection of Composites
}

\author{
Barbara Szymanik $^{1}$, Sreedhar Unnikrishnakurup ${ }^{2}$, Krishnan Balasubramaniam $^{2}$, Tomasz \\ ${ }^{1}$ Department of Electrical and Computer Engineering, \\ Faculty of Electrical Engineering, West Pomeranian University of Technology, \\ al. Piastów 17, 70-310 Szczecin, Poland \\ ${ }^{2}$ Centre for non-destructive Evaluation, Indian Institute of Technology Madras \\ Chennai 600 036, India \\ szymanik@zut.edu.pl
}

The studies on composite materials, due to their growing popularity in the industrial applications, are one of the most important branch of the non-destructive testing. The different kind of composites (ie. glass-fiber, carbon, with honeycomb or sandwich structures) are used in many industrial applications. The glass-fiber reinforced composites are widely used in the wind turbine blades and are exposed to various kinds of damages. The equipment reliability requirements force the development of accurate methods of their health monitoring. In this paper we present the numerical study of composite samples with artificial damages, using the active thermography with halogen lamp heating, connected with the fan cooling to obtain the heat sink effect.

Active infrared thermography is one of the methods that are widely used in the nondestructive testing of materials. The defects present in the internal structure of the tested material affect the free flow of heat. Sensitive infrared camera is used to observe the temperature distribution on the sample's surface. Using the halogen lamps as the heat source is one of the most convenient ways to provide the energy to the tested sample. In this method the heat is dissipated within the material with a rate dependent on the material properties. In case of composites the heat diffusion coefficient is small comparing to conductive materials. Therefore the heating rate is low and obtained results are difficult to processing and assessment. In this article the using of the fan cooling to obtain the heat sink effect is discussed. The fan cooling is used to cause the more rapid heat dissipation inside the material. This paper presents in detail the methodology and the results of the numerical analysis of the setup with combined heating sources and forced convective cooling. Moreover the results will be validated experimentally using chosen composite samples.

Acknowledgement

This work was supported in part by European Commission project HEMOW: Health Monitoring of Offshore Wind Farms (ref: FP7-PEOPLE-2010-IRSES-GA-269202).

Keywords: active infrared thermograpy, numerical analysis, composites, halogen lamps, heat sink effect 